$$
\text { "princz" — 2008/4/9 - 9:49 — page 293 — \#1 }
$$

\title{
Solving mathematical problems by using Maple factorization algorithms
}

\author{
MÁRIA PRINCZ
}

Abstract. Computer algebra gives methods for manipulating mathematical expression. In this paper we use the Maple software to solve some elementary problems. Computeraided approach in the instruction of mathematics helps to impart problem solving skills to students.

Key words and phrases: mathematics teaching, Maple, Computer Algebra Systems, factorization algorithms.

ZDM Subject Classification: C75, H05, H10, N85.

\section{Introduction}

In this note we would like to demonstrate the usefulness of the mathematical program packages, so-called Computer Algebra Systems (CAS) in solving certain hard elementary problems. Of course, the computers and programs do not substitute the intuitive mathematical thinking, however sometimes give some ideas for handling of problems. We work with one of the most popular mathematical softwares, the Maple 9.

\section{Examples}

Our first example comes from an old Russian mathematical olympiad.

Example 1. Prove that the number $2^{14}+5^{16}$ is composite.

Copyright (C) 2007 by University of Debrecen

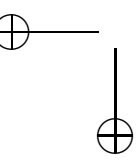




$$
\text { "princz" — 2008/4/9 - 9:49 — page 294 — \#2 }
$$

Using Maple, function ifactor, we have immediately that the prime factorization is

$$
x=2^{14}+5^{16}=380753 \cdot 400753 .
$$

One can see that the difference of two prime numbers is 20000 , so $380753=$ $390753-10000$ and $400753=390753-10000$. We obtain that

$$
2^{14}+5^{16}=390753^{2}-10000^{2} .
$$

This numerical result yields an idea for the mathematical solution of our first example. We search two integers $a$ and $b$, such that $x=2^{14}+5^{16}=b^{2}-a^{2}$. From this equality we have that

$$
b^{2}=x+a^{2}=\left(2^{7}\right)^{2}+\left(5^{8}\right)^{2}+a^{2} .
$$

Fortunately, the product $2 \cdot 2^{7} 5^{8}=10^{8}$ is a square, so it is clear that $b=2^{7}+5^{8}$ and $a=2^{4} \cdot 5^{4}$. We note that the exponents 14 and 16 are well chosen, since $2^{14}+5^{16}$ possesses only two large prime factors.

Next examples are advanced problems from the Mathematical Journal for Secondary Schools (KöMaL).

Example 2. (see KöMal F. 3107.) Prove that $1991^{1991}+1992^{1992}+\ldots+$ $1996^{1996}$ is not a square.

After a short calculation, Maple gives that

$$
1991^{1991}+1992^{1992}+\ldots+1996^{1996}=131 \ldots 87 .
$$

We note that this number has 6588 digits, however the Computer Algebra Systems can calculate on very large (500000 digits) integers. Using Maple command issqr we test if our number is square, and we obtain a negative answer.

We solve the problem without Maple. The last digit of a square number is 0 , $1,4,5,6,9$, thus our number is not a square. Now, in the mathematical proof we calculate the last digit of powers:

$$
\begin{array}{lll}
1991^{1991}=\ldots 1, & 1992^{1992}=\ldots 6, & 1993^{1993}=\ldots 3, \\
1994^{1994}=\ldots 6, & 1995^{1995}=\ldots 5, & 1996^{1996}=\ldots 6
\end{array}
$$

and we obtain the statement. 
Example 3. (see KöMal F. 3403.) Let $a, b, c$ be real numbers with $\frac{1}{a+b+c}=$ $\frac{1}{a}+\frac{1}{b}+\frac{1}{c}$. Prove

$$
\frac{1}{a^{5}+b^{5}+c^{5}}=\frac{1}{a^{5}}+\frac{1}{b^{5}}+\frac{1}{c^{5}}
$$

The function factor yields that

$$
\frac{1}{a+b+c}-\frac{1}{a}-\frac{1}{b}-\frac{1}{c}=-\frac{(a+b)(a+c)(b+c)}{a b c(a+b+c)}
$$

and

$$
\begin{aligned}
& \frac{1}{a^{5}+b^{5}+c^{5}}-\frac{1}{a^{5}}-\frac{1}{b^{5}}-\frac{1}{c^{5}}= \\
& -\frac{X\left(c^{4}-c^{3} b+c^{2} b^{2}-c b^{3}+b^{4}\right)\left(a^{4}-a^{3} b+a^{2} b^{2}-a b^{3}+b^{4}\right)\left(a^{4}-a^{3} c+a^{2} c^{2}-a c^{3}+c^{4}\right)}{\left(a^{5}+b^{5}+c^{5}\right) a^{5} b^{5} c^{5}}
\end{aligned}
$$

where $X=(a+b)(a+c)(b+c)$, and these factorizations show the mathematical proof of our problem. One can generalize this problem to other odd exponents instead of 5 . In this case Maple gives that

$$
\begin{aligned}
\frac{1}{a^{2 k+1}+b^{2 k+1}+c^{2 k+1}}- & \frac{1}{a^{2 k+1}}-\frac{1}{b^{2 k+1}}-\frac{1}{c^{2 k+1}}= \\
& -\frac{\left(c^{2 k+1}+b^{2 k+1}\right)\left(c^{2 k+1}+a^{2 k+1}\right)\left(a^{2 k+1}+b^{2 k+1}\right)}{\left(a^{2 k+1}+b^{2 k+1}+c^{2 k+1}\right) a^{2 k+1} b^{2 k+1} c^{2 k+1}}
\end{aligned}
$$

It is easy to see that

$$
(a+b)(b+c)(a+c)
$$

divides the numerator of

$$
\frac{1}{a^{n}+b^{n}+c^{n}}-\frac{1}{a^{n}}-\frac{1}{b^{n}}-\frac{1}{c^{n}}
$$

for $n=3,5,7,9,11, \ldots$.

We can use the function factor for solving similar exercises, especially for proving certain inequalities.

Example 4. (see KöMaL F. 3024.) Let $a, b, c$ be real numbers. Prove that

$$
(a b+b c+c a-1)^{2} \leq\left(a^{2}+1\right)\left(b^{2}+1\right)\left(c^{2}+1\right) .
$$




$$
\text { "princz" — 2008/4/9 — 9:49 — page 296 — \#4 }
$$

We have that

$$
\left(a^{2}+1\right)\left(b^{2}+1\right)\left(c^{2}+1\right)-(a b+b c+c a-1)^{2}=(a b c-a-b-c)^{2},
$$

and it con $\dot{Z} r m s$ the inequality.

ExAmple 5. (see KöMaL Gy. 3040.) Let $a, b$ be real numbers. Prove

$$
\frac{a^{4}+a^{2} b^{2}+b^{4}}{3} \geq \frac{a^{3} b+b^{3} a}{2} \text {. }
$$

The function factor works again, we get

$$
\frac{a^{4}+a^{2} b^{2}+b^{4}}{3}-\frac{a^{3} b+b^{3} a}{2}=\frac{1}{6}\left(2 a^{2}+a b+2 b^{2}\right)(b-a)^{2} .
$$

To prove the original inequality it is enough to show that $2 a^{2}+a b+2 b^{2} \geq 0$. A straightforward calculation gives $2 a^{2}+a b+2 b^{2}=\frac{(a+b)^{2}}{2}+\frac{3}{2}\left(a^{2}+b^{2}\right)$ which completes the proof. An alternative approach is using the command completesquare in the package student. It yields

$$
2 a^{2}+a b+2 b^{2}=2\left(a+\frac{b}{4}\right)^{2}+\frac{15 b^{2}}{8} .
$$

EXAMPLE 6. (see KöMaL B. 3856.) Let $a, b$ real numbers such that $a^{2}+b^{2}=$ $a^{2} b^{2}$ and $|a| \neq 1,|b| \neq 1$. Prove

$$
\frac{a^{7}}{(1-a)^{2}}-\frac{a^{7}}{(1+a)^{2}}=\frac{b^{7}}{(1-b)^{2}}-\frac{b^{7}}{(1+b)^{2}} \text {. }
$$

Using the function factor we have

$$
\begin{aligned}
\frac{a^{7}}{(1-a)^{2}}-\frac{a^{7}}{(1+a)^{2}}- & \frac{b^{7}}{(1-b)^{2}}+\frac{b^{7}}{(1+b)^{2}}= \\
& \frac{4(a+b)(a-b)\left(a^{2} b^{2}-a^{2}-b^{2}\right)\left(a^{4} b^{2}-a^{4}+a^{2} b^{4}-b^{4}\right)}{(a-1)^{2}(a+1)^{2}(b-1)^{2}(b+1)^{2}}
\end{aligned}
$$

which provides the proof.

\section{Conclusions}

Some examples of introducing Maple computer algebra software package as a tool in elementary problem solving is presented. Computer assisted instruction is gaining momentum in several disciplines. Computer-aided approach in the instruction of mathematics to impart problem solving skills to students. 


$$
\text { "princz" — 2008/4/9 - 9:49 — page } 297 \text { — \#5 }
$$

\section{References}

[1] Középiskolai Matematikai és Fizikai Lapok, http://www.komal.hu.

[2] Maple Student Center, http://www.adeptscience.co.uk/products/mathsim/maple/.

[3] R. M. Allen, T. Boustead and J. G. Mackenzie, Mathematical Power Tools - Maple, Mathematica, and Matlab versus Excel, Proc. th $^{\text {th }}$ AAEE Annual Convention and Conference, Melbourne, Australia, Dec 10-13, 1995, 89-92.

[4] W. E. Baylis, Theoretical Methods in the Physical Sciences: An introduction to problem solving using Maple, Birkhauser, Boston, 1994.

[5] W. Gander and J. Hrebicek, Solving problems in scientific computing using Maple and Matlab, Springer-Verlag, 1993.

MÁRIA PRINCZ

UNIVERSITY OF DEBRECEN

FACULTY OF TECHNICAL ENGINEERING

H-4024 DEBRECEN

ÓTEMETŐ UTCA $2-4$

HUNGARY

(Received February, 2006) 